\title{
Prácticas, ambientes y saberes: políticas de TIC en el contexto de la educación superior colombiana*
}

\author{
Practices, environments and knowledges: ICT Policies in the \\ context of the Colombian higher education
}

Recibido: 21 de febrero de 2012 - Revisado: 30 de mayo de 2012 - Aceptado: 17 de octubre de 2012

Julio Ernesto Rojas*

\section{Resumen}

Este artículo de revisión tiene como propósito presentar un estado de arte sobre la reflexión académica e investigativa producida en el contexto de la relación educación superior y políticas de TIC. Para lograr este propósito el texto se estructura en tres partes. Inicialmente se realiza un contexto histórico, breve, de las reflexiones y estudios de sistematización y análisis del tema. Dicho contexto se delimitó entre principios de los noventa y mediados del 2000. Posteriormente, se registra el estado de la cuestión en términos del planteamiento de problemas, metodologías empleadas, resultados obtenidos y discusiones abordadas. Finalmente se hacen algunas consideraciones a propósito de los resultados encontrados.

\section{Palabras clave}

Educación superior, política, investigación, Tecnologías de Información y Comunicación - TIC_-, globalización, competencias, sistemas triple hélice.

\footnotetext{
Abstract

This review article's has the purpose to show a state of the art on the academic and investigative reflection produced in the context of the relation: Higher Education and ICT policies. In order to achieve this purpose, the text is structured in three parts. Initially, a historic, brief context of the reflections, and systematization and analysis studies of the theme is accomplished. Said context was limited between the beginning of the nineties and mid 2000. At a later time, the state of the issue is registered in terms of the proposal of problems, used methodologies, obtained results and approached discussions. Finally, some considerations in relation to the found results are done.

Key words

Higher Education, Politics, Investigation, Information and Communication Technology - ICT, Globalization, Competences, Triple helix systems.
}

\footnotetext{
"Este artículo hace parte del trabajo de tesis doctoral titulado: Educación virtual: del discurso a las prácticas pedagógicas. Estudio etnográfico en tres instituciones de educación superior en Colombia, programa Teoría de la Educación y Pedagogía social, de la UNED-España. Fortalecido con el proyecto interinstitucional 'Políticas en TIC y Educación Superior, un análisis en relación con la institución educativa', adelantado durante el 2011 de manera conjunta entre los grupos de investigación Mundos virtuales - Mundos contemporáneos de la Universidad Santo Tomás, y Pedagogías, perteneciente a la Fundación Universitaria Los Libertadores. El proyecto se ejecutó con fondos de investigación de las dos universidades.

** Doctor en Teoría de la Educación y Pedagogía Social UNED-España. Estudios avanzados en Educación. Antropólogo Universidad Nacional de Colombia. Docente investigador de tiempo completo en el Departamento de Humanidades. Universidad Santo Tomás. Docente investigador en la Escuela de Posgrados de Comunicación de la Universidad Sergio Arboleda.

Correo electrónico:

juliorojas@usantotomas.edu.co

mesa.julio@gmail.com
} 


\section{Visión general del tema}

En Colombia, la reflexión sobre la relación entre educación y TIC surge en la década de los ochenta. Desde su primera etapa, delimitada hasta mediados de los noventa, el trabajo sobre TIC y educación se conoció bajo dos categorías: la Informática Educativa y la Educación a Distancia Virtual (Facundo, 2004; Parra Mosquera, 2010).

Diversos desarrollos han ocurrido desde esa época. Distintos estudios han documentado esta transformación (Maldonado y Maldonado, 2000; Salcedo Gómez, 2002; Rojas Mesa J., 2003). Estas iniciativas cumplieron el papel de motivar a instituciones e investigadores universitarios para contextualizar, fortalecer y formar diferentes corrientes de pensamiento sobre el tema (Maldonado G., 2010).

En Colombia se comienzan a pensar políticas de TIC a comienzos del 2000 con la formulación de la "agenda de conectividad" (MC y DNP, 2000). Esta fue el resultado del ordenamiento global en torno a la relación de las TIC con diferentes sectores de inversión social, entre los cuales se incluía la educación (Domínguez Figaredo, 2007; García A., Ruiz C., y Domínguez F., 2007; Facundo, 2008).

En el mundo universitario colombiano, la relación TIC-educación ha tenido una tendencia a desarrollar experiencias de corte más investigativo que docente. Muchas de ellas se han orientado a entender, desde diferentes perspectivas, la relación entre educación y TIC (Rueda y Quintana, 2004; Rueda, 2008). Ya no se discute la pertinencia de las TIC en el mundo educativo sino más bien cómo deben estar allí. Recientemente, esta relación ha tratado de abarcar los procesos de gestión de conocimiento con el fin de construir escenarios en la relación Universidad-EmpresaEstado (Maldonado Granados, Correa Hincapié y Alcócer Tocora, 2010).

Existen numerosas preocupaciones expresadas en la investigación Educación-TIC: la ausencia de una legislación más consistente sobre la educación virtual (Facundo, 2008); la prevalencia de una tendencia instrumental en los discursos, prácticas y ambientes que rodean la intervención de las TIC en el mundo educativo y, la escasa sensibilización y capacitación de comunidades educativas frente a la brecha digital. Esta última es vinculante con la brecha social de manera general; no obstante, en casos especializados como el universitario tiene que ver con barreras de idioma, acceso a bases de datos y repositorios de trabajos científicos, integración en redes de investigación y otros.

Este documento contribuye a la sistematización de muchas de esas experiencias investigativas recientes $\mathrm{y}$, a partir de sus resultados, describe algunas tendencias identificadas en las publicaciones consultadas.

\section{Resultados obtenidos}

Teniendo en cuenta los trabajos seleccionados, se identificaron cuatro líneas temáticas: 1. Autonomía y construcción de relaciones locales/globales a escala; 2. Educación, mercado y política; 3. Experiencias de implementación, y 4. Brecha social, de las cuales el mayor número de estudios se ubicó en educación, mercado y política (25), seguida de experiencias de implementación (19), autonomía y construcción de relaciones locales/globales a escala (12), y brecha social/brecha digital (9).

\section{Problema de investigación y método}

El objetivo general del proyecto consistió en adelantar un análisis de la relación entre educación superior y TIC, enfocándose para ello en el estudio etnográfico de tres instituciones de educación superior en Colombia, analizadas desde el enfoque teórico de los sistemas culturales (Rojas M. y Bernal, 2008; 2010). Este trabajo ha sido fortalecido con el desarrollo del proyecto interinstitucional 'Políticas en TIC y Educación Superior, un análisis en relación con la institución educativa', implementado entre la Universidad Santo Tomás y la Fundación Universitaria Los Libertadores. 
En ese sentido, se partió del supuesto de investigación de que existe una integración cada vez más compleja entre Educación Superior y Tecnologías de la Información y la Comunicación (TIC). Y que, para la universidad, este hecho obliga a pensar y construir discursos, prácticas y ambientes adecuados con el desarrollo contextual de dicha relación en una dimensión local/global, intercultural, interinstitucional e interdisciplinar.

\section{Tratamiento metodológico}

El tratamiento metodológico desarrollado para la investigación consistió en combinación entre el método etnográfico y el método arqueológico. Del primero, es decir del método etnográfico, se recurrió a tres técnicas: la observación etnográfica, la entrevista estructurada y el análisis documental. Del método arqueológico se utilizaron los tres procedimientos básicos que agrupan la caja de herramientas: descripción de las regularidades discursivas, descripción del enunciado y del archivo y la descripción arqueológica propiamente dicha, materializada en la redacción final de los resultados.

Para adelantar la revisión presentada aquí, se utilizó el método de análisis documental etnográfico y la descripción arqueológica. Este proceso tuvo como base la selección de documentos publicados en bases de datos especializadas y en revistas científicas, registrados como resultados de investigación, de revisión o reflexión, producto de estudios en el tema. Se registró una base inicial de 200 documentos, de los cuales finalmente se seleccionaron 65 como soporte a la revisión. Los criterios utilizados para su selección fueron la consistencia en la presentación de los resultados y el método utilizado en la investigación.

Para dar comienzo a la indagación se utilizaron los siguientes descriptores: educación superior, investigación, políticas, TIC y globalización. Se observaron trabajos realizados en América, Europa, Asía y África. Los datos fueron consignados en una matriz de referencia. Se utilizó el software Atlas-Ti con el fin de sistematizar y analizar la información en términos de enunciados y construcciones discursivas así como para visualizar mejor las relaciones identificadas.

Los documentos presentados fueron la base de análisis para la formulación de las cuatro líneas temáticas resultado de la revisión. Aunque la tabla 1 expone la clasificación de los documentos en relación con cada línea, se advierte que en el desarrollo del trabajo algunos documentos son citados en más de una de ellas. A continuación, se presenta la clasificación documental realizada:

Tabla 1

Cuadro de organización de las fuentes documentales según las líneas temáticas identificadas

\begin{tabular}{|c|l|lc|}
\hline \multicolumn{3}{|c|}{ ORGANIZACIÓN DE DOCUMENTOS REVISADOS POR LÍNEA TEMÄTICA Y PAÏS } \\
\hline Línea temática & Autor & País & Año \\
\hline & Alejandro Artopoulos y Débora Kozak & Argentina & 2011 \\
& Rosa Mariana Melo & Venezuela & 2008 \\
AUTONOMÍA Y & Claudio Rama & Argentina & 2009 \\
CONSTRUCCIÓN DE & Jos Belen & Holanda & 2011 \\
RELACIONES LOCALES/ & Stavros Moutsios & Dinamarca & 2010 \\
GLOBALES A ESCALA & Marck Bray & Francia & 2009 \\
& Barbara Coelho Neves & Brasil & 2010 \\
& Jesús Alberto Andrade Castro, & Venezuela & 2007 \\
\hline
\end{tabular}




\begin{tabular}{|c|c|c|c|}
\hline \multicolumn{4}{|c|}{ ORGANIZACIÓN DE DOCUMENTOS REVISADOS POR LÍNEA TEMÄTICA Y PAÏS } \\
\hline Línea temática & Autor & País & Año \\
\hline & Alejandro Uribe Tirado & Colombia & 2010 \\
\hline & Montse Guitert, Teresa Romeu & España & 2009 \\
\hline & Kwame Akyeampong & Ghana & 2009 \\
\hline \multirow{27}{*}{$\begin{array}{c}\text { EDUCACIÓN, MERCADO } \\
\text { Y POLÍTICA }\end{array}$} & Claudio Rama & Argentina & 2007 \\
\hline & Javier Hermo, Cecilia Pitelli & Argentina & 2008 \\
\hline & Ana Tamayo Salcedo & Colombia & 2009 \\
\hline & Jocelyne Gacel-Avila & México & 2007 \\
\hline & Ana Claudia Rozo Sandoval & Colombia & 2010 \\
\hline & Rocio Rueda Ortiz & Colombia & 2008 \\
\hline & Raquel Goulart Barreto & Brasil & 2008 \\
\hline & Raquel Goulart Barreto, Roberto Leher & Brasil & 2008 \\
\hline & Esther Hermes Luck & Brasil & 2009 \\
\hline & Joan Anton Sánchez, Cristina Alonso, & & \\
\hline & Alejandra Bosco & Chile & 2008 \\
\hline & daño Veloso, Eduardo Quiroga Aguilera & Chile & 2009 \\
\hline & Ángel Facundo & Colombia & 2009 \\
\hline & $\begin{array}{l}\text { Luis Facundo Maldonado, Víctor Uribe, } \\
\text { Adriana Lizcano, Juan Sequeda, }\end{array}$ & & \\
\hline & Eliécer Pineda & Colombia & 2008 \\
\hline & Edel Serrano Iglesias & Colombia & 2008 \\
\hline & Linda Alejandra Leal Urueña & Colombia & 2009 \\
\hline & Francesc Esteve & España & 2009 \\
\hline & Francisca Cruz, Eloiza Oliveira & Brasil & 2010 \\
\hline & Mario Barajas, Gloria Gannaway & España & 2007 \\
\hline & Diego Ernesto Leal Fonseca & Colombia & 2008 \\
\hline & Löfström Erika, Anne Nevgi & Finlandia & 2007 \\
\hline & Marta Quiroga Lobos & Nueva Zelanda & 2008 \\
\hline & Jairo Castillo Cortes & Colombia & 2009 \\
\hline & Eric Meyer, William Dutton & Inglaterra & 2009 \\
\hline & Rosemary Agbonlahor & Nigeria & 2008 \\
\hline & Javier Peña Sánchez & Colombia & 2010 \\
\hline \multirow{13}{*}{$\begin{array}{l}\text { EXPERIENCIAS DE } \\
\text { IMPLEMENTACIÓN }\end{array}$} & Natasa Tomic & Serbia & 2009 \\
\hline & Robert Meurant & Corea & 2011 \\
\hline & Evangelina Zepeda & México & 2008 \\
\hline & Leonel Corona & Uruguay & 2011 \\
\hline & Enrique Saravia & México & 2011 \\
\hline & Juan Cristóbal Cobo Romaní & Reino Unido & 2009 \\
\hline & Paloma Antón Arés & España & 2010 \\
\hline & Niklas Hanes; Sofia Lundberg & Suecia & 2008 \\
\hline & $\begin{array}{l}\text { Narciso Cerpa, Andres Ruiz, Carolina } \\
\text { Cabrera, Pamela Hadweh, Fabián Vergara }\end{array}$ & Chile & 2007 \\
\hline & $\begin{array}{l}\text { Pedro Gomez, Maria Cañadas, } \\
\text { Camilo Soler, Ángela Restrepo }\end{array}$ & Colombia & 2012 \\
\hline & $\begin{array}{l}\text { Deiby Montenegro, Manuel Menéndez } \\
\text { Centro de Investigación en }\end{array}$ & Colombia & 2009 \\
\hline & Telecomunicaciones -Cintel- & Colombia & 2010 \\
\hline & Gabriel Valerio, Jaime Ricardo Valenzuela & México & 2011 \\
\hline
\end{tabular}




\section{ORGANIZACIÓN DE DOCUMENTOS REVISADOS POR LÍNEA TEMÄTICA Y PAÏS}

\begin{tabular}{|l|l|lr|}
\hline Línea temática & Autor & País & Año \\
\hline \multirow{5}{*}{} & Katrina Meyer & Estados Unidos & 2009 \\
& María Martínez & Colombia & 2011 \\
& Elias Said Hung, Carlos Arcila Calderón, & Colombia & 2012 \\
& Luis Núñez & Reino Unido & 2007 \\
& Neil Selwyn & Reino Unido & 2008 \\
& Eynon Rebecca & Nueva Zelanda & 2006 \\
\hline \multirow{5}{*}{ BRECHA SOCIAL/ } & Eduardo Aguado López, Rosario Rogel & & \\
& Salazar, Arianna Becerril García, & & 2009 \\
& Graciela Baca Zapata & España & 2011 \\
& Juan Carlos Torres Díaz, Alfonso Infante & & \\
& Moro & Ecuador & 2010 \\
& Iván Ramírez Pinzón; Alejandro & & 2010 \\
& Gutiérrez & Colombia & 2011 \\
& Ancízar Narváez Montoya & Colombia & 2012 \\
& Jean-Claude Guédon & México & 2011 \\
& Sandra Miguel, Nancy-Diana Gómez, & & \\
& Paola Bongiovani & Argentina & 2012 \\
& Jorge Enrique Delgado Troncoso & Argentina & 2007 \\
\hline & Edwin Montoya Munera, Malgorzata & & Colombia \\
& Lisowska, Dago Hernando Bedoya Ortiz & Espana & \\
& José Álvarez Castillo & &
\end{tabular}

\section{Discusión y presentación de los resultados de investigación}

El contexto de la educación superior y las políticas de TIC, discutido aquí, se soporta en una reflexión sobre el sentido amplio de lo político. Amplio en el contexto de las sociedades del conocimiento y la información y su necesaria interacción en escenarios locales y globales.

Existe un enfoque monopolio en el mundo digital; el sujeto se transforma en nodo de red. Su prioridad es el aprendizaje permanente y la gestión personal de un conocimiento que debe diferenciar de la información (Rama, 2009). Finalmente, debe proyectarse como un producto autogestionado en el mercado digital (Castells M., 2008; Bauman Z., 2010).

En el mundo universitario este panorama es interrogado. ¿El sujeto es libre y autónomo en la red, tal y cómo enuncian estas teorías del prosumer?, ¿cómo debe pensar el sistema educativo este fenómeno?
Se puede afirmar que lo político define en sí mismo el conjunto de tensiones construidas como confluencia de múltiples intereses. El poder entendido como las acciones que enfrentan acciones e intereses de otros para consensuar una idea de mundo, de sociedad, de conocimiento, de cultura, de derecho. Lo político no es el conjunto de leyes instauradas en una determinada sociedad, sino la jurisprudencia que deviene sobre la aplicación de dichas leyes, los acontecimientos que crea (Deleuze G., 1996; Restrepo, 2008). Presumiblemente, las formas de ejercer autonomía en el sentido educativo están asociadas con una tendencia que alude a relaciones entre sujeto-saber-poder y la manera como estas se agencian en los diferentes sistemas culturales de las sociedades humanas (Sánchez Amaya, 2010; Londoño Vásquez y Frias Cano, 2011).

Las interacciones locales y globales a escala de las sociedades y sus múltiples microsistemas se soportan cada vez más en estructuras de redes informáticas y telecomunicativas. Es- 
tas narrativas atraviesan permanentemente los sistemas culturales universitarios al punto de que muchos de sus desarrollos e implementaciones son pensados cada vez con más articulación y vinculación a los ambientes digitales (Adel y Ludovick, 2008). De estas y otras experiencias de orden local y global se ocupan los trabajos revisados y analizados aquí.

\section{Autonomía y construcción de relaciones locales/globales a escala}

Los trabajos aquí registrados expresan algunas de las contradicciones, tensiones, dificultades y también posibilidades que acontecen en torno a la comprensión de la relación entre lo local y lo global con su consecuente impacto en el mundo de la educación superior.

Los estudios regionales - como parte de los trabajos que componen este apartado- confirman el valor estratégico de la educación en cuanto motor de cambio social e impulsor del desarrollo. Asociada con la incorporación de las TIC — a través de políticas, programas y proyectos - se erige como alternativa de transformación social, política, económica y cultural.

Dos de las investigaciones referidas a Latinoamérica evidencian la irrupción de instancias distintas a los Ministerios de Educación para alcanzar estos propósitos: se registran cambios en el modo de gestión gubernamental de la educación y en los procesos de digitalización de la educación, caracterizados por la intervención de nuevas agencias de gobierno, así como de otros actores sociales, económicos y tecnológicos que antes no hacían parte de la comunidad educativa (Artopoulos y Kozak, 2011).

Adicionalmente, en cada país las condiciones socio-técnicas inciden en el modelaje pedagógico organizacional de las experiencias de incorporación de TIC en el aula. Sobre el particular, Melo (2008) señala que los investigadores e investigadoras de la región tienen el convencimiento de que con sus trabajos pueden contribuir a resolver problemas sociales y que en términos de fragmentación y glocalización es indispensable "colocarnos al lado de una globalización alternativa y no de una globalización homogenizadora" (p. 118).

La investigación sobre la Comunidad Iberoamericana y Europea (Belén, 2011) revisa las implicaciones de la globalización en la educación. Señala la importancia de profundizar los vínculos y programas compartidos en materia de educación para promover los procesos de integración regional, consolidar las relaciones entre los miembros de la Comunidad Iberoamericana y fortalecer sus conexiones con la Unión Europea. Hasta el momento, dicha relación fluye entre una baja participación del grueso de las universidades de América Latina con respecto al diseño de procesos sobre la internacionalización de la educación superior y la escasa respuesta regional latinoamericana. Para Rama, las condiciones tecno-económicas y geopolíticas de América Latina requieren que se piense el tema sobre un nuevo concepto: "el bien público internacional" (2009).

Al respecto, Moutsios (2010) elabora un análisis global sobre lo político en la relación globalización económica y política transnacional de la educación. Argumenta que el poder no se queda exclusivamente en los que producen el discurso educativo dominante ni tampoco en su reproducción dentro de las legislaciones nacionales, las políticas locales y prácticas pedagógicas. Además, se proyecta con el fortalecimiento de una percepción específica y local de aquello que la educación debe ser, que para la época actual parece haberse centrado exclusivamente en el incremento de la competitividad económica, el crecimiento económico y finalmente un desarrollo afianzado por muchos y entendido por muy pocos.

Los trabajos reseñados de Francia (Crossley, Packer y Bray, 2009) y Brasil (Coelho Neves, 2010) permiten observar cómo el desplazamiento de políticas particulares en ma- 
teria de educación, inclusión digital y tecnologías hacia el diseño de estrategias globales están articulados a fenómenos de competitividad económica en el plano educativo. Lo anterior hace necesario pensar los conceptos de ciudadanía y cohesión social.

El trabajo de (Andrade Castro y CampoRedondo, 2007) en Venezuela plantea que la educación se mantiene anclada en un ambiente ideológico endogámico que la ha ido 'elitizando' y enfatizando las relaciones jerárquicas y de desigualdad. Adicional a esto, las TIC han enfatizado un determinismo tecnológico que provoca desconfianza sobre el uso asignado a ellas en políticas nacionales y locales.

En Colombia, los trabajos de Facundo (2008; 2009) identifican una doble condición en el desarrollo de la política de TIC en Colombia. En primer lugar, el cambio entre las modalidades educativas con mediación tecnológica fue rápido y brusco (desde el sistema postal hasta el virtual), hecho que no permitió un manejo teórico/metodológico adecuado de las mismas, y en segundo lugar, el desarrollo normativo sobre el tema ha avanzado bruscamente y con muchas rupturas, impidiendo que se consolide una política coherente en los desarrollos educativos con mediación tecnológica.

Resalta la importancia de los sistemas de información digital, ya que han permitido reescribir diversos mitos. Uno de ellos es la presunción (desmentida en las estadísticas) de una alta deserción adjudicada a los sistemas educativos con alta mediación tecnológica en comparación con los presenciales (2009).

Rojas (2012) describe el proceso que ha realizado el Gobierno colombiano para integrarse en los índices y rankings internacionales para la medición de las TIC. Revela que la última década fue funesta para la aplicación de TIC en educación. El diseño e implementación de políticas para la integración y manejo de TIC fue casi nulo y el país terminó perdiendo pues- tos en las listas internacionales. No obstante, el desarrollo en dotación de infraestructura y acceso general a internet ha sido rápido $\mathrm{y}$ expansivo a una gran parte del territorio nacional. Según Uribe Tirado (2010), el Gobierno colombiano ha enfocado todos sus esfuerzos en la infraestructura pero no en la alfabetización ni en la formación de competencias informacionales.

Romeu y Guitert (2009) proponen pensar una universidad que responda a la cultura digital emergente y que trascienda los esquemas educativos habituados a la repetición. Según los autores, se debe generar innovación basada en procesos y competencias, desde perspectivas interdisciplinares de educación expandida. Hay que considerar la alfabetización digital y sus consecuentes formas de representación de conocimiento apoyadas en los recursos de internet social. En esencia, formulan alternativas que contribuyan a integrar los procesos educativos en los esquemas socio-tecno-comunicativos propios de los entornos digitales. Finalmente, Akyeampong (2009) advierte que en Ghana (África) la reforma para introducir nuevas TIC exige cambios curriculares en la formación docente, ya que la ausencia de relación entre política y práctica educativa desarticula a las IES del contexto, con las consecuencias que ello implica en las ofertas educativas.

\section{Educación, mercado y política}

Las investigaciones agrupadas en esta categoría evidencian una fuerte tendencia entre educación superior y mercado. En consecuencia, describen el contexto de intereses, políticas, estrategias e impactos, producto de la orientación de la educación superior en términos de mercado.

Así por ejemplo, criterios de reducción de costos, formación en serie, gestión de calidad, franquicias educativas, internacionalización de la educación, educación transnacional y transfronteriza, eficiencia, competencia, modelos triple hélice de articulación entre empresa-uni- 
versidad-Estado, competitividad y eficacia, se consolidan como objetos del discurso del nuevo ordenamiento educativo.

Los estudios de Rama (2007), Hermo y Pittelli (2008) en Argentina y Tamayo (2009) en Colombia advierten sobre el enfoque economicista de las reformas educativas y las políticas trazadas desde organismos internacionales como el Banco Mundial, el Banco Interamericano de Desarrollo (BID), la Comisión Económica para América Latina y el Caribe (Cepal). $\mathrm{Su}$ materialización se presenta en acuerdos como el AGCS (Acuerdo General sobre el Comercio de Servicios, o en inglés: General Agreement on Trade in Services - GATS-) y en otros acuerdos de libre comercio, bilaterales o multilaterales que redefinen la educación superior como un servicio económico que puede permitir el lucro de quienes intervienen en su producción y/o gestión.

El modelo empresarial emplaza al sistema educativo. Dicha alineación de lo educativo bajo el prisma empresarial origina reformas e innovaciones educativas en todas las esferas de la vida económica, política, cultural y social (Tamayo Salcedo, 2009).

Esta configuración propicia el surgimiento de un mercado educativo transnacional que responde a la consolidación cada vez más evidente y cotidiana de una pequeña aldea-mercado constituida, no por sujetos de derecho, sino por usuarios de servicios (Rama, 2009). Este autor describe esta situación como "(...) una nueva modalidad educativa, que tiene escalas diferenciadas, que no tiene fronteras, que permite un fraccionamiento de los procesos educativos localizando en un país las tutorías, en otro los softwares o hardwares, en otro los alumnos o los profesores, en una lógica que permite una educación supranacional" (2007, p. 33). En consecuencia, las universidades pequeñas, en ámbitos locales, se ven obligadas a generar mecanismos para su reestructuración con miras a crear alianzas de acceso al mercado educativo global y a diseñar planes de negocios para mantenerse en el mercado educativo local.

Sin embargo, surgen otros enfoques que abogan por una combinación de los paradigmas mencionados. Según Gacel-Ávila (2007) se puede transformar el paradigma educativo transnacional mencionado por uno de educación internacional y humanista. En los ámbitos locales y nacionales de América Latina, una educación humanista no riñe con presupuestos de sostenibilidad. Finalmente, considera que una práctica educativa cuyo objeto sea el fomento de la diversidad cultural, el respeto a la identidad y a la diferencia, con un escenario de apoyo en TIC-Educación, es altamente benéfica para el desarrollo de estos países.

De otro lado, los estudios mencionados se complementan con análisis de segmentos más específicos como la docencia. Existen numerosas investigaciones preocupadas por el papel del docente. En algunas de ellas este se identifica como estratégico; en otras, se estudian los diseños e implementaciones de programas internacionales y nacionales que promueven su formación en materia de TIC.

Algunos de los trabajos realizados en Colombia (Rozo Sandoval, 2010; Rueda Ortiz, 2008) describen la necesidad analizar con más detenimiento problemas fundamentales como la identidad, la subjetividad, las interacciones y su relación con el conocimiento, la información y el poder, ya que son aspectos que se imbrican cada vez más en una continuidad entre los ambientes físicos y digitales; "de este modo se alejan de la idea que confronta y antagoniza estos dos entornos" (Rozo Sandoval, 2010, p. 41).

Las investigaciones registradas en Brasil sobre el tema (Goulart Barreto, 2008; Goulart Barreto y Leher, 2008; Luck, 2009) refieren una vinculación entre formación docente-educación a distancia y TIC, desde donde se reconoce la dimensión política ideológica en la inserción de las tecnologías en educación y se produce 
un cambio significativo en los roles asignados a quienes participan en esta relación, así el tutor - desprovisto de su carácter docente- reemplaza al profesor y es contratado en condiciones más desfavorables; los materiales y quienes participan de su diseño y producción se posicionan de manera significativa en esta relación. Adicionalmente, emergen otras figuras encargadas de la administración y gerencia del servicio.

Los resultados de las investigaciones de Chile y España ratifican la importancia de la formación de profesores y advierten que los discursos optimistas descuidan otros componentes del sistema como la necesidad de efectuar reformas curriculares (Sancho M., Ornellas, Sánchez, Alonso y Bosco, 2008) y la posibilidad de contar con observatorios para la gestión del conocimiento en educación (Careaga Butter, Avendaño Veloso y Quiroga Aguilera, 2009). Para Careaga y otros, el funcionamiento del observatorio virtual se encuentra soportado en redes de contactos y colaboración. Surge como una perspectiva distinta e innovadora en el campo de la formación docente y permite abordar los estudios sobre la comunidad académica en perspectiva de sistemas culturales. Finalmente argumentan que este tiene una inmensa posibilidad de transformar capital intelectual en capital social.

Una de las líneas estratégicas que presenta relaciones más fuertes entre educación y mercado es la educación mediada digitalmente (e-learning, b-learning, m-learning, educación a distancia virtual). Estos esquemas representan un riesgo en el manejo de la proporción del número de estudiantes por curso. Usualmente algunas instituciones han caído en él (Facundo, 2009).

Por otro lado, los dispositivos a través de los cuales se "moderniza" y amplía el portafolio digital de la institución educativa (objetos virtuales, ambientes virtuales soportados en redes sociales, tableros inteligentes, en general industria de contenidos y dispositivos) ubican al estudiante en el centro del proceso. Con ello, muchas de esas propuestas pretenden impulsar modelos de gestión de conocimiento local (Maldonado, Uribe O., Lizcano, Sequeda y Pineda, 2008; Maldonado Granados y Serrano Iglesias, 2008; Maldonado Granados y Leal Urueña, 2009; Esteve, 2009), estiman la importancia del conocimiento en una sociedad que enuncia estar basada en él (Correa, Matos, Cruz y Oliveira, 2010), aprecian las posibilidades de la convergencia tecnológica para disponer ambientes educativos enriquecidos (Barajas y Gannaway, 2007; Maldonado Granados y Leal Urueña, 2009) y promueven el diseño de materiales como oportunidad nacional e internacional para ser capitalizada dentro de procesos de procesos de producción de ambientes y contenidos (Leal Fonseca, 2008).

En este sentido, la investigación realizada en la Universidad de Helsinki, Finlandia, reconoce el valor de la planeación estratégica en los procesos de implementación de TIC y refiere como son utilizadas para la enseñanza en todas las facultades e institutos, pero no se informa sobre mejoras en la calidad, lo que implica desafíos importantes para la pedagogía (Érika y Nevgi, 2007). El estudio efectuado en Irlanda, Nueva Zelanda y Chile mostró que las políticas de inserción de TIC en esos países disponen de órganos administrativos para tal efecto. Desde allí se promueven los planes, programas y estrategias que orientan estos propósitos, y vía los coordinadores tecnológicos se procura el éxito de las mismas (Quiroga Lobos, 2008). Las experiencias adelantadas en Colombia permitieron inicialmente evidenciar un potencial para la construcción de objetos de aprendizaje para ambientes digitales y proyectar una línea de mercado internacional sobre el tema (Leal Fonseca, 2008; Castillo Cortés, 2009).

Finalmente, en este conjunto se identificaron tres investigaciones que indagan por aspectos epistemológicos y filosóficos que se dan en las relaciones entre educación y tecnología. Así, el trabajo de Oxford que explora la e-research 
y su implicación en la realización de trabajos interdisciplinares, indaga por el uso de la red en perspectiva filosófica. Para ello, define cuatro expectativas teóricas: investigación por cohortes, políticas metodológicas, certeza-consumo de verdad y experiencias tecnológicas (Meyer T. y Dutton, 2009). El estudio en Nigeria, África, indaga por las actitudes de los universitarios frente al uso de las TIC en procesos de docencia e investigación y sugiere tener en cuenta las particularidades de la cultura institucional de cada universidad. También sugiere tener en cuenta la perspectiva de género en los procesos de sensibilización (Agbonlahor, 2008). Y el trabajo de Peña en Colombia (Peña Sánchez, 2010) indaga por la condición filosófica de la virtualidad en los procesos de educación superior.

\section{Experiencias de implementación}

En el análisis inicial de los artículos correspondientes a esta clasificación se observa como las ideas asociadas a bienestar, inclusión, mejoramiento en la calidad de vida y educación, posibilidades de desarrollo y expectativas de producción de conocimiento se mantienen como los ejes sobre los cuales se llevan a cabo procesos de implementación de políticas en materia de TIC y educación.

En el estudio sobre las experiencias en Serbia es notoria la referencia a mejoras educativas y a la calidad de vida mediante el uso de TIC (Tomic, 2009) en tanto que el trabajo de Corea sobre alfabetización digital, utilizando diferentes dispositivos tecnológicos, hace énfasis en el progreso del aprendizaje de lenguas no nativas por medio de las mediaciones tecnológicas (Meurant, 2010). Para la experiencia mexicana, el éxito en la implementación está dado por las alianzas entre educación - sector productivo y Estado (Zepeda, 2008). En una línea más regional, el trabajo de García y Muñoz se pregunta por el lugar de las regiones en el nuevo ordenamiento económico y social promovido en el escenario global de la sociedad de la información y del conocimiento. Parale- lamente propone una afirmación de principios de solidaridad y colaboración interinstitucional para abordarlo (García y Muñoz, 2010).

Por otro lado, Corona señala que "una de las variables centrales de los posibles nuevos caminos es el uso productivo del conocimiento, lo cual comprende un conjunto de interrelaciones institucionales complejas de los conocimientos técnicos, aprendidos con la práctica; los tecnológicos, que se nutren de la ciencia, y los científicos" (Corona, 2011, p. 182).

En el escenario de posibilidades, pero también de desafíos, las políticas de alfabetización digital, inclusión y accesibilidad constituyen elementos prioritarios que precisan ser revisados en función de algunos aspectos. Lo inadecuado y complejo que implican las alfabetizaciones universalistas. Consecuentemente, el énfasis de las diferencias significativas entre países y regiones. El análisis en Brasil (Saravia, 2009) refiere la importancia de la participación de la comunidad como elemento decidor y ejecutor para minimizar los factores de fracaso en este proceso. El estudio sobre desarrollo de e-competences para la Unión Europea analiza el impacto de las políticas y programas de alfabetización digital en el aprendizaje de los estudiantes y propone redefinir el concepto de e-competences en función de la creación de la "fuerza de trabajo competente" (Cobo, 2009).

El trabajo de Antón (2010) aborda el tema de accesibilidad en la universidad española y evidencia la necesidad de generar programas y estrategias específicas de inclusión tanto a la educación superior, como al mundo laboral, para las personas en condición de discapacidad, en cumplimiento con políticas internacionales y nacionales sobre el tema (Antón Arés, 2010); en tanto que en Suecia, el interés por vincular a los segmentos de la población excluidos de la educación superior se resuelve vía ofertas educativas mediadas tecnológicamente, para el caso e-learning, y las preguntas que se formulan para dar continuidad a esta política (ley de 
2001) se relacionan con la cobertura, la calidad comparada con la formación presencial y la inversión en términos de bienestar (Niklas y Lundberg, 2008).

En las temáticas alusivas a e-learning, educación virtual, desarrollo de contenidos y usos de internet, las investigaciones reflejaron inquietudes diversas que van desde el grado de conectividad de las instituciones educativas y su proyección en el espacio de la red (Cerpa, Ruiz, Cabrera, Hadweh y Vergara, 2007), los proceso de producción y evaluación de contenidos digitales (Gómez, Cañadas, Soler y Restrepo, 2009; Montenegro y Menéndez, 2009) hasta el estado del crecimiento del mercado para identificar el papel que Colombia podría desempeñar (Leal Fonseca, 2008; Cintel, 2010). La identificación de condiciones para el trabajo colaborativo online y la constitución de los contactos en redes sociales como repositorios de información ha sido trabajado en México (Valerio y Valenzuela, 2011) el reconocimiento de las transformaciones en el sector educativo generadas por la primera universidad virtual de los Estados Unidos (UAA), creada en 1995 (Meyer, 2009), y cerrando este grupo de trabajo, el análisis sobre los retos que enfrenta la educación superior en la sociedad de la información: ofrecer un conocimiento oportuno y práctico, verdaderamente útil a los estudiantes y profesionales, y cómo el e-learning, el m-learning, el b-learning y el u-learning se convierten en sistemas ventajosos para cumplir este propósito (Martínez, 2011).

En Colombia, se detalla una variabilidad constante en la política de los planes de formación de docente en TIC (Rueda O., 2009), así como grandes dificultades en la consolidación de trabajos colectivos interinstitucionales o la implementación de redes para este efecto, a pesar de los múltiples intentos por crear dichas redes y escenarios colaborativos (Hung, Arcila Calderón y Núñez, 2012).

Finalmente, tres investigaciones con enfoques más críticos llaman la atención sobre la necesidad de observar en profundidad los cambios reales que tienen lugar tanto a nivel cognitivo como sociocultural y económico en los espacios de formación mediados tecnológicamente. El trabajo realizado en el Reino Unido muestra cómo los usos más generalizados de las TIC se construyen en términos limitados, lineales, rígidos y alejados de la creatividad y productividad tan referidas por los tecnólogos de la educación. Frente a esto, los investigadores proponen volver sobre las reflexiones del pensamiento contemporáneo y propender por construcciones que trasciendan los usos periféricos de los instrumentos (Selwyn, 2007).

La investigación en Inglaterra sobre la incorporación real de las tecnologías en la vida escolar y cotidiana de los usuarios de un "campus virtual" concluyó que el potencial de las TIC para algunos aspectos de enseñanza y aprendizaje se presenta limitado y que no parece estar transformando radicalmente la educación en términos de las expectativas que sobre el tema se refieren (Rebecca, 2008). Cierra este grupo de trabajos el artículo de Gibbons (2006), que analiza la problemática asociada al desarrollo de políticas de inserción de TIC y las dificultades de su incorporación en las aulas. Los investigadores realizan un análisis crítico del discurso y observan la cultura de la institución escolar, encuentran cómo las expectativas sobre los educadores impulsadas por las políticas y por la industria que provee la tecnología no corresponden a los resultados finales. En respuesta, sugiere la necesidad de desarrollar estrategias e investigaciones que permitan identificar las condiciones reales y complejas de esta relación.

\section{Brecha social / brecha digital}

Los trabajos ubicados en este ítem (Uribe Tirado, 2010) (Aguado López, Rogel Salazar, Becerril García y Baca Zapata, 2009) (Torres Díaz e Infante Moro, 2011) (Ramírez Pinzón y Gutiérrez, 2010) (Narváez Montoya, 2010) (Goulart Barreto y Leher, 2008) (Rueda O, 
2009) (Cintel, 2008) reflejan las inquietudes de los investigadores en relación con las múltiples y diversas formas de distanciamiento de los sujetos con las TIC.

En una perspectiva general, la brecha digital tradicional persiste. Algunos análisis de los índices de medición muestran que la concentración de riqueza e iniquidad social son causas primordiales para el acceso a las tecnologías (Ramírez Pinzón y Gutiérrez, 2010). En relación con los imaginarios construidos socialmente, el estudio de Ramírez y Gutiérrez analiza cómo el efecto conjunto de las herramientas metodológicas de la brecha digital determina el comportamiento en el uso y apropiación de las TIC. En tanto, se ratifica el papel protagónico de la educación como escenario estratégico a través del cual se proponen políticas, planes y programas para minimizar los factores de exclusión de la sociedad contemporánea. En este sentido, las investigaciones advierten sobre las dificultades reales de los países que no forman parte de las economías dominantes para ingresar en condiciones de equidad al mercado del conocimiento (Narváez Montoya, 2010).

El estudio realizado por Cintel en Colombia plantea la urgencia de analizar la "brecha digital" como conjunto y consecuencia de las "brechas sociales" tradicionales (2008), conclusión que es corroborada por los estudios de Torres e Infante en las universidades ecuatorianas, quienes concluyen que "el nivel de ingresos del núcleo familiar del estudiante incide en los usos e intensidad de uso de las herramientas de internet, por tanto existe una diferenciación o brecha que se ajusta a la realidad socioeconómica" (2011, p. 87). En Brasil, el trabajo de Goulart y Leher afirma que esta brecha digital está altamente dirigida por los intereses de organismos internacionales como el Banco Mundial. Estos organismos construyen escenarios de nuevas realidades a través del condicionamiento de las políticas en los Estados nacionales y la creación de neologismos. Los discursos y sus prácticas materializan las nuevas expresiones de sujeto que necesita el mercado global (2008).

En este contexto, es muy adecuado el trabajo realizado por Rueda en Colombia, quien pregunta por el sujeto y el ejercicio de la ciudadanía (2009). El estudio de Goulart y Leher concluye que la figura del Estado Nación ha entrado en un proceso de transformación radical. Se interroga por las relaciones en el ejercicio de la ciudadanía como expresión de las conexiones socio-tecno-culturales surgidas en los espacios digitales. En su análisis, plantea la necesidad de trascender los dualismos (tecnófobos/tecnófilos) y de enfrentar las políticas que orientan el uso y manejo formal e 'instruccional' de las tecnologías y sus contradicciones con aquellas que incentivan procesos de creación y producción, y más aún, con su uso como contrapoder a sistemas que intentan hegemonizar al sujeto.

Por otro lado, en los espacios universitarios ha venido surgiendo un escenario más complejo de la brecha digital, relacionado con la dinámica de funcionamiento e impacto de las listas mundiales sobre resultados de investigación y publicaciones científicas. El trabajo de Aguado y otros analiza los más importantes de estos rankings entre los que se encuentran Shanghai Jiao Tong University Ranking y The Times World University Ranking. En Iberoamérica, Scimago (2009).

Según estos autores, las nuevas tecnologías favorecen la publicación en línea y la visibilidad inmediata de resultados de investigación; sin embargo, ha generado una brecha mayor en tanto el valor de la investigación está determinada por el lugar en dichos rankings: "No basta con publicar los resultados de los estudios, el movimiento de acceso abierto permite contar con mayor y mejor presencia dentro de la red -además de cumplir con las funciones asignadas a las instituciones de educación superior-, criterio importante en el momento de evaluar a las instituciones en este tipo de rankings (...)" (2009, p. 14). 
Este panorama es complementado por Guedon, quien formula una división entre ciencia principal y ciencia periférica que solo es posible fracturar con el movimiento de acceso abierto. Su análisis de la manera en que Brasil ha adelantando un proceso de reducción de barreras de socialización de la producción científica con la creación de la biblioteca electrónica Scielo, le permite concluir que el acceso abierto es un movimiento altamente beneficioso para los países emergentes en la lucha por validar su producción científica (2011).

Lo anterior ha motivado otra serie de movimientos y estudios. Los trabajos de Miguel y Bongiovani, en Argentina, plantean una ruta metodológica interesante ya que, recogiendo las rutas "dorada y verde" presentadas para alcanzar el acceso abierto en la declaración de la BOAI (Budapest Open Access Initiative -2001-), generan un esquema que permite comparar resultados entre países y campos temáticos sobre la producción del conocimiento en los ámbitos de acceso abierto (2012). En Colombia, el trabajo que viene realizando Uribe Tirado sobre el análisis de políticas de "inclusión y alfabetización informacional" permite concluir que estas aún responden exclusivamente a criterios generales de lo que se entiende por "brecha digital", es decir, infraestructura en conectividad y conexión de redes físicas (Uribe Tirado, 2010). En un plano regional, el trabajo de Delgado (2011) resalta el impresionante crecimiento que han tenido en las últimas dos décadas las revistas arbitradas de América Latina. El autor de este estudio realiza una clasificación que arroja resultados sobre la participación de los países de América Latina en repositorios de acceso abierto. El estudio registra una participación avanzada de Colombia, al menos por el número de publicaciones presentes en las bases de datos. A ello han contribuido experiencias como BDCOL; Proyecto de Biblioteca Digital Colombiana, creado en el 2007 con la participación inicial de 13 universidades de las cuales se mantienen 11 (Montoya Munera, Lisowska y Bedoya Ortiz, 2011).
Desde una visión más prospectiva, la investigación de Castillo observa los procesos de convergencia en la educación en el contexto de la sociedad red, que de acuerdo con su estudio, "en el futuro próximo, las necesidades de homologación de los aprendizajes planteadas por las exigencias de los mercados darán lugar, con el soporte de plataformas tecnológicas, a un entramado de redes que favorecerá una convergencia aún mayor de la educación del siglo XXI” (Álvarez Castillo, 2007, p. 23).

\section{Sobre lo metodológico, hallazgos y tensiones}

Lo metodológico en las investigaciones analizadas evidenció un interés, de la comunidad, en desarrollar perspectivas compresivas de problematizaciones que en sí misma son complejas: las relaciones entre educación - tecnología - política, en el marco de un escenario de economía global que cada vez más impulsa criterios homogeneizadores y universalistas, los cuales son trasladados al escenario de observación. Aquí es importante enfatizar que si bien la mayoría de los estudios se caracterizaron por abordajes interpretativos, interdisciplinares para comprender las múltiples conexiones entre los temas, también se registraron trabajos de tipo descriptivo que - en la línea de propuestas de gobierno o institucionales - comunicaron los alcances de los mismos, sus expectativas y beneficios.

Para los investigadores de la temática, el análisis crítico del discurso, los análisis cruzados, los estudios descriptivos-interpretativos constituyeron las opciones metodológicas más empleadas, solo un número muy reducido de artículos registraron el uso de opciones metodológicas volcadas a una sola disciplina. Los estudios regionales e internacionales (para los casos de análisis que contrastan experiencias de dos o más países, pero que no necesariamente componen una perspectiva regional) advierten la necesidad de considerar las especificidades de los países en función de las culturas diversas 
y las condiciones socio - económico - tecnológicas que no necesariamente facilitan la uniformidad en los datos.

Este componente descrito en las consideraciones metodológicas emerge como uno de los factores más reiterativos de las tensiones identificadas a saber:

- Entre la homogenización y estandarización de las políticas, y la diversidad de los saberes, culturas y formas de producción de conocimiento (global / local).

- Instalación de nuevas tecnologías con prácticas pedagógicas más especializadas.

- Las expectativas tecno-utópicas frente a los resultados en el sistema.

- La tensión de intereses al interior del sistema cultural universitario a saber: la formación docente en aspectos especialmente académicos frente a la institución educativa proyectada en una relación condicionada por los intereses de la empresa y el mercado.

- Los bajos índices de inversión en procesos de formación e investigación universitaria frente a las altas expectativas de gobiernos y empresarios con relación a los resultados en procesos de gestión de conocimiento.

- La creciente presión por socializar los resultados de la producción científica en repositorios digitales con acreditación y condiciones de alto impacto.

\section{Conclusiones}

La relación entre política-educación-TIC se advierte a primera vista contextualizada en una noción homogénea de globalización. Sin embargo, a medida que se ahondó en los pro- blemas de investigación revisados se encontró una tensión diversa y con desarrollos desiguales entre lo local y lo global. Esta diversidad se vio reflejada en los impactos y resultados analizados.

Como resultado de lo anterior, se describen varios aspectos en donde se encontró el desarrollo de la relación política-educación superior y TIC.

Un primer aspecto tiene que ver con la construcción de sentidos de autonomía. Esta concuerda con la manera como se construyen las relaciones entre lo local y lo global. Aunque pareciera haber un consenso en la noción de lo local, no ocurre lo mismo con lo global. Este último es entendido de diferentes maneras; algunos de los trabajos analizados lo presentan como algo exterior a los sistemas locales, relación que se expresa por ejemplo en una directa interferencia de organismos internacionales y transnacionales, en políticas y orientaciones nacionales y locales, hecho que deja un escaso, por no decir nulo, margen al ejercicio de autonomía en los sistemas locales.

Por el contrario, otras investigaciones entienden lo global como una agrupación a escala particular con proyección general. Los resultados de estos trabajos plantean la emergencia de iniciativas de carácter global, a escala, como respuesta a iniciativas globales, a escala, que manejan una doble condición: están diseñadas en arquitectura, acceso y funcionamiento sobre condiciones locales y específicas pero sus intereses e impactos se proyectan en términos de una cobertura global y homogeneizante. Es el caso de los rankings en ámbitos especializados de la investigación universitaria.

Frente al establecimiento de monopolios de repositorios digitales, revistas y bases de datos excluyentes han surgido iniciativas también globales como el "acceso abierto" con características incluyentes, amplias y reguladas. Esta alternativa para la socialización de la producción científica es especialmente benéfica para los países emergentes. 
Esta tensión deja entrever que lo global es básicamente una agrupación de sistemas locales que se cruzan con sistemas similares. Ninguna de estas agrupaciones es necesariamente totalizante y frente a este discurso universalista surgen alternativas que lo confrontan. El caso del movimiento de "acceso abierto" es significativo de esta situación.

En segundo lugar, los estudios evidencian una relación cada vez más estrecha entre educación superior-mercado-política. Se describe el desplazamiento sistemático de un paradigma de ciudadano con derechos hacia uno de usuario con servicios, así como el protagonismo que tienen las TIC en dicho proceso.

El impacto en la educación superior de esta transformación de paradigmas es significativo. Las críticas sobre una intervención económica y de mercado en la universidad, que tradicionalmente se habían centrado una posibilidad de masificación de la educación a través de plataformas digitales, encuentran más elementos de configuración de este paradigma de mercado y educación.

Los trabajos reseñados registran la crisis de un sistema educativo tradicional territorializado, unificado y administrado nacionalmente. En su lugar, advierten la consolidación de un sistema de educación transnacional, transterritorial e internacional, con un perfil económico global. Con una nueva batería de conceptos, este sistema habla de criterios de eficiencia, eficacia, calidad, desterritorialización, descentralización, tercerización y mercado. Todo lo anterior, con vistas a un mundo global y de mercado, se sostiene en un principio de competitividad.

La universidad se ve obligada a medirse permanentemente en el contexto global y a presentar indicadores acordes con los criterios mencionados. En todo este panorama, las TIC parecen ser una bisagra central que conecta el pequeño sistema cultural universitario con un mundo global a escala cuyos bordes deben ser transitados por la gestión del conocimiento.

\section{Referencias}

Adel, Y. B., \& Ludovick, R. (2008). Uses of Information and Communication Technologies in Europe's Higher Education Institutions: From Digital Divides to Digital Trajectories. Revista Universidad de la sociedad del conocimiento, 5(8).

Agbonlahor, R. (2008). Individual Characteristics as Correlates of Attitudes to Information Technology among Nigerian University Lecturers. African Journal of Library, Archives and Information Science, 18(2), pp. 131-146.

Aguado López, E., Rogel Salazar, R., Becerril García, A. y Baca Zapata, G. (2009). Presencia de universidades en la Red: la brecha digital entre los Estados Unidos y el resto del mundo. (U. O. -UOC-, Ed.). Revista de Universidad y Sociedad del Conocimiento -RUSC-. Recuperado de: http://redalyc.uaemex.mx/ pdf/780/78011179002.pdf

Akyeampong, K. (2009). Las tecnologías multimedia y reforma educativa en África: el caso de Ghana. Comunicar, XVI(32), pp. 109-118.

Álvarez Castillo, J. L. (2007). Los procesos de convergencia de la educación en el contexto de la sociedad red. Revista Española de Pedagogía, 2369, pp. 5-25.

Andrade Castro, J. A. y Campo-Redondo, M. S. (2007). Tecnologías de información para la inclusión digital. Apertura, 7(006), pp. 63-75.

Antón Arés, P. (2010). Programas y apoyos técnicos para favorecer la accesibilidad en la universidad. Apertura, 10, pp. 1-13. 
Artopoulos, A. y Kozak, D. (agosto de 2011). Estilos de adopción de tecnología en la educación latinoamericana. Revista Iberoamericana de Ciencia, Tecnología y Sociedad: Recuperado de: http://www. revistacts.net/index.php?option $=$ com_sec tionexyview $=$ categoryyid $=27 \mathrm{yItemid}=98$

Barajas, M. y Gannaway, G. (Julio-octubre de 2007). Implementing E-learning in the traditional higher educations institutions, jul-oct 2007. Education Resources Information Center -ERIC-. Recuperado de: http://www.informaworld. com/openurl?genre $=$ articleyid $=\mathrm{d}$ oi:10.1080/03797720701840609

Bauman, Z. (2010). Mundo consumo. Ética del individuo en la aldea global. Barcelona: Paidós.

Belén, J. (2011). La internacionalización en casa en una perspectiva global: un estudio crítico del Informe del 3.er Estudio Global de la AIU. (n. 2.-1. Revista de Universidad y Sociedad del Conocimiento (RUSC),. 8.). Globalización e internacionalización de la educación superior [monográfico en línea]: Recuperado de: http://rusc.uoc.edu/ojs/index.php/rusc/article/view/v8n2-beelen/v8n2-beelen

Careaga Butter, M.; Avendaño Veloso, A. y Quiroga Aguilera, E. (2009). Observatorio virtual basado en un modelo pedagógico de gestión del conocimiento para innovar en educación. Innovación Educativa, 9(47), pp. 73-90.

Castells, M. (2008). Comunicación, poder y contrapoder en la sociedad red. Los medios y la política. Revista Telos, 74, enero-marzo. Recuperado de: http://sociedadinformacion.fundacion.telefonica.com/url-direct/pdf-generator?tipoContenido $=$ articul oyidContenido $=2009100116310137$
Castillo Cortés, J. (25 de julio de 2009). Los tres escenarios de un objeto de aprendizaje. (O. d. Iberoamericanos, Ed.). Revista Iberoamericana de Educación: Recuperado de: http://www.rieoei.org/2884.htm

Cerpa, N.; Ruiz, A.; Cabrera, C.; Hadweh, P. y Vergara, F. (2007). Evaluación del nivel de adopción de internet en las universidades chilenas en base al modelo emica. Ingeniare, pp. 270-282.

Cintel. (2008). Brecha digital en Colombia. Bogotá: Cintel.

Cintel. (2010). Un acercamiento a la industria de contenidos digitales. Estudio cualitativo: Casos de empresas en Colombia. Bogotá: Cintel.

Cobo, J. (2009). Strategies to promote the development of E-competences in the Next Generation of professionals: European and international trends. Reino Unido: Flacso-México.

Coelho Neves, B. (2010). Análise das políticas de informação: sociedade da informação com foco na inclusão digital do global ao local. Revista Iberoamericana de Ciencia, Tecnología y Sociedad - CTS: Recuperado de: http://redalyc.uaemex.mx/src/inicio/ArtPdfRed. jsp?iCve $=92414779008$

Corona, L. (2011). Prospectiva tecnológica para América Latina. En Unesco, América Latina y el Caribe: escenarios posibles y políticas sociales. (pp. 181 - 207). Montevideo, Uruguay: Unesco.

Correa, R.; Matos, E., Cruz, F. y Oliveira, E. (2010). Reflexões sobre três programas de política educacional con uso de tecnologías en um Brasil integrado. Varia Scientia, 09 (16), pp. 11-22. 
Crossley, M., Packer, S. y Bray, M. (2009). Education in the Small States of the Commonwealth: Towards and Beyond Global Goals and Targets. The Round Table, 98(405), pp. 731-751.

Deleuze, G. (1996). Conversaciones 19721990. Valencia: Pre- textos.

Delgado Troncoso, J. E. (2011). Papel del acceso abierto en el surgimiento y consolidación de las revistas arbitradas en América Latina y el Caribe. (Iesal-Unesco, Ed.). Educación Superior y Sociedad. Recuperado de: http://ess.iesalc.unesco.org.ve/ index.php/ess/article/viewFile/408/346

Domínguez Figaredo, D. (2007). Devenir histórico de mitos y usos tecnológicos en educación a distancia: de la lectoescritura al e-learning. Textos de la Cibersociedad, 10. Recuperado de: http://www.cibersociedad.net/textos/articulo.php?art=136.

Érika, L. \& Nevgi, A. (2007). From strategic planning to meaningful learning: diverse perspectives on the development of webbased teaching and learning in higher education. British Journal of Educational Technology, 38 (2), March 2007, pp. 312324.

Esteve, F. (05 de 2009). Bolonia y las TIC: de la docencia 1.0 al aprendizaje 2.0. La Cuestión Universitaria, boletín electrónico de la Cátedra Unesco de Gestión y Política Universitaria. Universidad Politécnica de Madrid. Recuperado de: http://www. lacuestionuniversitaria.upm.es/web/articulo.php?id_articulo=42

Facundo, A. (2009). Análisis sobre la deserción en la educación superior virtual en Colombia: el caso de la UNAD-Colombia. Revista de Investigaciones UNAD, 8 (2), pp. 117-149.
Facundo, A. (2008). La educación superior a distancia en Colombia: un análisis del marco normativo. En C. Rama, M. Mena y A. Facundo. El marco regulatorio de la educación superior a distancia en América Latina y el Caribe (pp. 153199). Bogotá: Ediciones Hispanoamericanas.

Facundo, A. (2004). La educación virtual en Colombia. En C. Rama, La eduación virtual en América Latina, situación y perspectivas (pp. 165-217). Chile: Unesco.

Gacel-Ávila, J. (agosto de 2007). Calidad y educación sin fronteras. Apertura, revista de innovación educativa (U. d. Guadalajara, Ed.). Recuperado de: http://www. udgvirtual.udg.mx/apertura/num6/pdfs/ calidad_fronteras.pdf

García, A., L.; Ruiz, C, M. y Dominguez, F., D. (2007). De la educación a distancia a la educación virtual. Madrid: UNED.

García, Y. y Muñoz, L. (2010). La educación superior centroamericana en contexto de construcción de espacios comunes del conocimiento. Actualidades investigativas en educación, 10, pp. 1-28. Univ. de Costa Rica.

Gibbons, A. N. (enero de 2006). The politics of technology inj early childhood in Aotearoa / New Zeland. Australian Journal of Early Childhood. Recuperado el 15 de julio de 2011, de http://goliath.ecnext.com/ coms2/gi_0199-6152061/The-politicsof-technology-in.html

Gómez, P.; Cañadas, M. C.; Soler, C. y Restrepo, Á. M. (2009). FUNES: un repositorio digital para públicar y compartir documentos en educación matemática. Jornadas de investigación en el aula de matemáticas:dimensión histórica, social y 
cultural de las matemáticas. Universidad de los Andes. Recuperado de: http://funes.uniandes.edu.co/1792/

Goulart Barreto, R. (2008). As Tecnologías na política nacional de formacão de professores a distãncia: entre a expansão e a redução. Educación Social, pp. 919-937.

Goulart Barreto, R. y Leher, R. (2008). Do discurso e das condicionalidades do B.M., a educação superior emerge "terciária". Revista Brasileira de Educação, 13(39), pp. 423-436.

Guédon, J. C. (2011). El acceso abierto y la división entre ciencia "principal" y "periferica" (C. L. -Clacso-, Ed.) Crítica y emancipación. Revista Latinoamericana de Ciencias Sociales, 6, pp. 135-180.

Hermo, J. y Pitelli, C. (2008). Globalización e internacionalización de la Educación Superior. Apuntes para el estudio de la situación en Argentina y el Mercosur (UNED, Ed.) Revista Española de Educación comparada 14, pp. 243-268.

Hung, E. S.; Arcila Calderón, C. y Núñez, L. (enero-junio de 2012). La promoción de la E-investigación en Colombia. El caso del Centro Virtual de Altos Estudios en Altas Energías (Cevale2). E-colabora. Revista de ciencia, educación, innovación y cultura apoyadas por Redes de Tecnología Avanzada, pp. 131-144.

Leal Fonseca, D. E. (noviembre de 2008). Iniciativa colombiana de objetos de aprendizaje: situación actual y potencial para el futuro (U. D. Guadalajara, Ed.). Apertura. Revista de Innovación Educativa. Recuperado de: http://www.udgvirtual. udg.mx/apertura/num8/pdfs/iniciativa colobiana.pdf
Londoño Vasquez, D. y Frias Cano, L. (2011). Análisis crítico del discurso y arqueología del saber: dos opciones de estudio de la sociedad. Palabra Clave, 14 (1), pp. 101-121.

Luck, E. H. (marzo de 2009). El proceso de transformación tecnológica y la formación docente (U. O. Catalunya, Ed.) Revista de la sociedad del conocimiento UOC. Recuperado de: http://rusc.uoc.edu/ojs/index. php/rusc/article/view/v6n1-luck

Maldonado, L. F. y Maldonado, P. I. (2000). Nuevas tecnologías aplicadas a la educación. Estado del arte de la investigación 1990-1999. Nuevas tecnologías aplicadas a la educación. Estado del arte de la investigación. 1990-1999 (pp. 143-203). Bogotá: ICFES.

Maldonado, L. F.; Uribe O., V.; Lizcano, A.; Sequeda, J. y Pineda, E. (2008). Comunidades de aprendizaje mediadas por redes informáticas (U. d. Sabana, ed.). Revista educ.educ. Facultad de Educación. Universidad de la Sabana, 11(1), pp. 199-224.

Maldonado Granados, L. F. y Serrano Iglesias, E. (2008). Construcción de una red de aprendizaje. Nomadas, pp. 211-222.

Maldonado Granados, L. F. y Leal Urueña, L. A. (2009). Análisis de interacciones en foros y chat: consolidación de grupo y liderazgo cmunicativo en un curso de lógica matematica. Revista Iberoamericana de Educación a Distancia, 12(2), pp. 189210.

Maldonado Granados, L. F. y Leal Urueña, L. A. (2009). Sincronía en la comunicación pedagógica y heterogeneidad de los grupos respecto al nivel de conocimientos previos: efectos en el aprendizaje en aulas digitales (U. P. nacional, ed.). Tecne Episteme y Didaxis, 25, pp. 8-21. 
Maldonado G, L. F. (julio de 2010). Editorial. $X$ Congreso Nacional de Informática Educativa. Recuperado de: http://www. ribiecol.org/ribie_a/cd/index.html

Maldonado Granados, L. F., Correa Hincapié, N. y Alcócer Tocora, M. (2010). Los Centros de Gestión de Conocimiento Especializados: una estrategia de desarrollo desde las regiones. Revista de Investigaciones Universidad Nacional abierta y a Distancia-UNAD-, 9(3), pp. 85-107.

Martínez, M. (2011). Experiencias de inclusión educativa en Colombia: hacia el conocimiento útil. Revista de Universidad y Sociedad del Conocimiento (RUSC), 12.

MC, M. D. y DNP, D. N. (2000). Documento. CONPES 3072. 'Agenda de conectividad: el salto a internet'. Bogotá: DNPMinisterio de Comunicaciones.

Melo, R. M. (2008). Educación y multilateralismo en América Latina: El tema educativo en la cumbre de las Américas. Revista de Pedagogía. Universidad Central de Venezuela. Recuperado de: http://redalyc.uaemex.mx/pdf/659/65908404.pdf

Meurant, R. C. (2010). iPad Table Computing to foster Korean EFL Digital Leteracy. Recuperado de: http://web.me.com/ rmeurant/INSTITUTE/HOME.html.

Meyer T, E. y Dutton, W. H. (20 de agosto de 2009). Experience with New Tools and Infrastructures of Research: An Exploratory Study of Distance From, and Attitudes Toward, e-Research. Prometheus, Critical Studies in Innovation. Recuperado de: http://www.tandfonline.com/doi/ abs/10.1080/08109020903127802

Meyer, K. (2009). Western Governors University: creating the first virtual university.
New Directions for Higher Education, 46, pp. 35-43.

Miguel, S.; Gómez, N.-D. y Bongiovani, P. (2012). Acceso abierto real y potencial a la producción científica de un país. El caso argentino. El profesional de la información, 21(2), pp. 146-153.

Montenegro, D. y Menéndez, M. (2009). Evaluación Sistemas de Metabúsqueda para BDCOL. Bogotá: Ministerio de Educación Nacional, Instituto Colombiano para el Desarrollo de la Ciencia y la Tecnología, Red Nacional Académica de Tecnología Avanzada.

Montoya Munera, E., Lisowska, M., y Bedoya Ortiz, D. H. (julio-diciembre de 2011). Surgimiento de iniciativas de acceso abierto (Renata, ed.). E-Colabora. Revista de ciencia, educación, innovación y cultura apoyadas por Redes de tecnología avanzada. Recuperado el 15 de abril de 2012, de http://publicaciones.renata. edu.co/index.php/RCEC/article/viewFile/57/pdf

Moutsios, S. (2010). Power, politics and transnational policy-making in education ( $\mathrm{T}$. D. Department of Education, ed.). Globalisation, Societies and Education, 8(1), pp. 121-141.

Narváez Montoya, A. (2010). Educación, capitalismo y desarrollo: cultura alfabética y globalización anglosajona. Signo y Pensamiento, XXIX (57), pp. 248-267.

Niklas, H. \& Lundberg, S. (2008). E-learning as a regional policy tool: principles for a cost-benefit analysis. $R U$ y SC. Revista de Universidad y Sociedad del Conocimiento, 5 (1), pp. 12-21.

Parra Mosquera, C. A. (2010). Interseccioines entre las TIC, la educación y la pedagogía 
en Colombia. Hacia una reconstrucción de múltiples miradas. Nómadas. Revista de investigación de la Universidad Central, 33, pp. 215-225.

Peña Sánchez, J. (2010). La concepción filosófica de lo virtual en la educación virtual. Revista colombiana de Educación, 58, pp. 118-139.

Quiroga Lobos, M. (2008). Análisis comparado de experiencias de introducción de las TIC en el aula. El rol de coordinador tecnológico y su impacto en el éxito de las políticas públicas. REICE. Revista Electrónica Iberoamericana sobre Calidad, Eficacia y Cambio en educación, 6(4), pp. 149-164.

Rama, C. (11-12 de mayo de 2009). La internacionalización ante el bien público en América Latina: entre las ideas y las realidades. Recuperado de: http://www. claudiorama.name/sites/default/files/1-2 $\mathrm{La}$ internacionahttp://www.claudiorama. name/ensayos/lización de la educación superior y el bien público.pdf

Rama, C. (01 de agosto de 2007). La despresencialización de la educación superior en América Latina: ¿tema de calidad, de cobertura, de internacionalización o de financiamiento?. Recuperado de: http:// www.claudiorama.name/sites/default/ files $/ 2-4 \% 20 \mathrm{La} \% 20$ despresencializacion $\% 20 \mathrm{de} \% 201 \mathrm{la} \% 20$ educacion $\% 20$ superior $\% 20 \mathrm{en} \% 20 \mathrm{Am} \% \mathrm{C} 3 \% \mathrm{~A} 9$ rica $\% 20$ Latina.\%20tema\%20de\%20calidad, $\% 20$ cobertura, $\% 20$ internacionalizacion $\% 20$ o\%20de\%20financiamiento.pdf

Ramírez Pinzón, I., y Gutiérrez, A. (2010). Brecha digital en Colombia. Bogotá: Cintel.

Rebecca, E. (2008). The use of the world wide web in learning and teaching in higher education. Innovations in Education and Teaching International, 45(1), pp. 15-23.
Restrepo, E. (2008). Cuestiones de método: "eventualización" y problematización en Foucault. Tabula Rasa, 8, pp. 111-132.

Rojas Mesa, J. (2003). La educación a distancia en Colombia, una historia que vale la pena contar. En J. Rojas m, y W. Ortiz R, Ideas para integrarse a la educación a distancia (pp. 105-116). Bogotá: UNAD.

Rojas M, J. y Bernal, C. (2008). Etnografía aplicada en ambientes digitales: una experiencia metodológica en dos proyectos de doctorado en el contexto de la educación superior en Colombia. Hallazgos. Revista de la Unidad de Investigación de la Universidad Santo Tomás, 9, pp. 167-189.

Rojas Mesa, J., y Bernal Granados, C. (2010). Análisis de los ambientes digitales en la educación superior desde una perspectiva de virtualidad: avances metodológicos de investigación en dos proyectos de doctorado. En G. I. Reyes Corredor, Filosofía y cultura en Colombia y América Latina. Memorias del IV Encuentro Tomasino de Investigadores. Julio de 2008 (pp. 225263). Bogotá, Colombia: Universidad Santo Tomás (USTA).

Rojas Mesa, J. E. (2012). Politicas públicas en TIC y educción superior: un análisis en relación con la institución educativa. Bogotá: Universidad Santo Tomás.

Romeu, T. y Guitert, M. (febrero de 2009). A digital literacy proposal in online Higher Education: the UOC scenario (U. O. Catalunya, ed.). Department of Computer Science, Multimedia and Telecommunication Universitat Oberta de Catalunya, UOC. Recuperado de: http://www.elearningeuropa. info/files/media/media18503.pdf

Rozo Sandoval, C. (2010). Dimensión pedagógica de la educación virtual: una reflexión pendiente (U. P. Nacional, ed.). Pedago- 
gía y saberes. Facultad de Educación, 32, pp. 33-44.

Rueda, R., y Quintana, A. (2004). Ellos vienen con el chip incorporado. Bogotá: Universidad Central /Universidad Distritial Francisco José de Caldas.

Rueda, R. (2008). Cibercultura: metaforas practicas sociales y colectivos en red. Nomadas, 8-20.

Rueda O, R. (2009). Convergencia tecnológica: síntesis o multiplicidad política y cultural (U. Javeriana, ed.). Signo y pensamiento, XXVIII(54), pp. 114-131.

Rueda Ortiz, R. (enero-junio de 2009). Convergencia tecnológica: síntesis o multiplicidad política y cultural (S. y pensamiento y r. d. Javeriana, eds.) Colección Convergencia tecnológica: síntesis o multiplicidad politica y cultural. Recuperado de: http://recursostic.javeriana.edu.co/ cyl/syp/components/com booklibrary/ ebooks/5408.pdf

Rueda Ortiz, R. (2008). Ciberculturas, capitalismo cognitivo y cultura (Universidad de Barcelona, ed.). Temps d'Educació, 34, pp. 251-264.

Salcedo Gómez, F. (2002). Historia de Internet en América Latina y el Caribe. Colombia: historia de la conexión de Uniandes a Internet. Recuperado de: http://interred.wordpress. com/2002/05/12/colombia-historia-de-laconexion-de-uniandes-a-internet-2/

Sanchez Amaya, T. (2010). La caja de herramientas como opción metodológica. Análisis, 76, pp. 71-102.

Sancho M, J.; Ornellas, A.; Sánchez, J. A.; Alonso, C. y Bosco, A. (2008). La formación del pofesorado en el uso educativo de las TIC, una aproximación desde la política educativa. Praxis educativa, pp. 10-22.
Saravia, E. (2009). La participación de la comunidad en la implementación de políticas públicas. Caso: las políticas de alfabetización y de inclusión digital en Minas Gerais, Brasil. En F. Martiñez Navarro y V. Garza Cantú, Política pública y democracia en América Latina. Del análisis a la implementación (pp. 243-253). México.

Selwyn, N. (2007). The use of computer technology in university teaching and learning: a critical perspective. Journal of Computer Assisted Learning, pp. 83-94.

Tamayo Salcedo, A. L. (2009). El discurso de la transformación de la universidad, entre la universalidad y la particularidad. Universidad Autónoma del estado de México. Recuperado de: http://redalyc.uaemex.mx/src/inicio/ArtPdfRed. jsp?iCve $=193414420004$

Tomic, N. M. (2009). Education for the future - case of Serbia. The juridical current journal. Año XII,38(3).

Torres Díaz, J. C. e Infante Moro, A. (2011). Desigualdad digital en la universidad: uso de internet en Ecuador. Comunicar. Revista cientifica de Educomunicación, XIX (37), pp. 81-88.

Uribe Tirado, A. (2010). Estado de arte de la alfabetización informacional en Colombia. Edimburgo: Federación Internacional deAsociaciones e Instituciones Bibliotecarias (IFLA).

Valerio, G. y Valenzuela, J. R. (2011). Contactos de redes sociales en línea como repositorios de información (UOC, ed.). Revista de Universidad y Sociedad del Conocimiento (RUSC), 8(1), pp. 128-141.

Zepeda, E. (2008). Políticas públicas y educación superior en el marco de la sociedad del conocimiento. Virtual Educa, p. 12. Zaragoza. 
